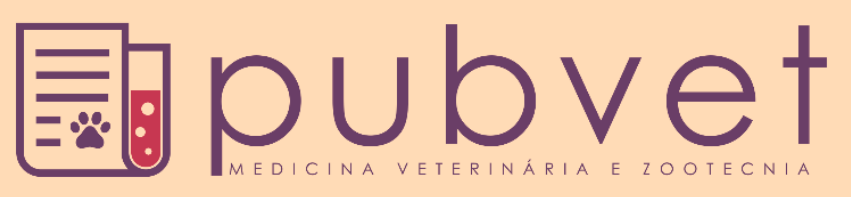

https://doi.org/10.31533/pubvet.v14n1a483.1-5

\title{
Emprego de IATF como alternativa para melhorar a eficiência reprodutiva de vacas leiteiras
}

\author{
Cicero Antônio Sobreira Fidelis ${ }^{1 *} \bullet$, Darllan Philippe Palitot Fernandes ${ }^{2} \bullet$ \\ 1*Professor do Instituto Federal do Pará. Conceição do Araguaia-PA Brasil. \\ ${ }^{2}$ Extencionista da Empresa de Assistência Técnica e Extensão Rural do Estado do Pará. Conceição do Araguaia-PA Brasil. \\ *Autor para correspondência,E-mail: cicero.sobreira@bol.com.br.
}

\begin{abstract}
Resumo. O período de serviço é o tempo decorrido entre o parto e a concepção, o intervalo médio desejado é de 85 a 115 dias, quando prolongado resulta em prejuízos como, queda na produção e diminuição do número de crias na vida produtiva da fêmea bovina. $\mathrm{O}$ objetivo deste estudo foi demonstrar a produtores da agricultura familiar que através da Inseminação Artificial em Tempo Fixo (IATF) é possível reduzir o período de serviço e consequentemente o intervalo entre partos, melhorando assim a eficiência reprodutiva do rebanho. Foram selecionadas 10 propriedades da agricultura familiar, no município de Conceição do Araguaia, sudeste paraense, distante $991 \mathrm{~km}$ da capital do Estado. Foram utilizadas 100 vacas mestiças/multíparas de baixa produção, palpadas para confirmação de vazias e a pelo menos 45 dias de paridas, referência baseada nas anotações encontradas em cadernos nas propriedades. No Dia 0 (implante intra-vaginal de progesterona e $2 \mathrm{ml}$ de benzoato de estradiol intramuscular), Dia 8 (retirada do implante mais $2,5 \mathrm{ml}$ de prostaglandina, $1,5 \mathrm{ml}$ de Gonadotrofina Coriônica Equina e $0,5 \mathrm{ml}$ de cipionado de estradiol, intramuscular), Dia 10 a inseminação. O diagnóstico de gestação foi feito aos 60 dias após a inseminação com ultrassom, as vacas diagnosticadas vazias foram encaminhadas ao repasse com touros das respectivas propriedades. A taxa de concepção contabilizada foi baseada em 94 vacas, já que 03 vacas vieram a óbito e 03 foram encaminhadas ao abate antes do diagnóstico de gestação. Obteve-se uma taxa de concepção de 53\% (50/94), destas, 60\% (30/50) estavam paridas entre 45 a 60 dias, enquanto $40 \%$ (20/50) estavam paridas a mais de 120 dias. Conclui-se que a IATF reduz o período de serviço e consequentemente o intervalo entre partos, demonstrando utilidade também em rebanhos da agricultura familiar, podendo resultar em maior possibilidade de produção/ano de leite e bezerros.
\end{abstract}

Palavras chave: período de serviço, prenhez, reprodução

\section{Use of IATF as an alternative to improve the reproductive efficiency of dairy cows}

Abstract. The period of service is the time between calving and conception, the desired average interval is 85 to 115 days, when prolonged results in losses such as decreased production and decreased number of calves in the productive life of the female bovine. The aim of this study was to demonstrate to family farmers that through Fixed Time Artificial Insemination (IATF) it is possible to reduce the period of service and consequently the interval between births, thus improving the reproductive efficiency of the herd. Ten family farms were selected in the municipality of Conceição do Araguaia, southeastern Pará, 991 $\mathrm{km}$ from the state capital. One hundred crossbred/multiparous cows of low production were palpated to confirm voids and at least 45 days of calving. On Day 0 (intravaginal progesterone implant and $2 \mathrm{ml}$ intramuscular estradiol benzoate), Day 8 (implant 
withdrawal plus $2.5 \mathrm{ml}$ prostaglandin, $1.5 \mathrm{ml}$ Equine Chorionic Gonadotropin and $0.5 \mathrm{ml}$ estradiol cipionate, intramuscular), Day 10 the insemination. The pregnancy diagnosis was made 60 days after ultrasound insemination, the empty diagnosed cows were sent to bulls with their respective farms. The conception rate was based on 94 cows, since 03 cows died and 03 were slaughtered before the pregnancy diagnosis. A conception rate of 53\% (50/94) was obtained, of which 60\% (30/50) were calved between 45 to 60 days, while 40\% (20/50) were calved for more than 120 days. It can be concluded that the TAI reduces the period of service and consequently the interval between births, also demonstrating utility in family farming herds, which may result in a greater possibility of milk / calf production / year.

Keywords: period of service, pregnancy, reproduction

\section{IATF como alternativa para mejorar la eficiencia reproductiva de las vacas lecheras}

Resumen. El período de servicio es el tiempo entre el parto y la concepción, el intervalo promedio deseado es de 85 a 115 días, cuando prolongado resulta em perjuicios como pérdidas em la producción y disminución del número de terneros en la vida productiva de la hembra bovina. El objetivo de este estudio fue demostrar a los agricultores familiares que a través de la Inseminación Artificial de Tiempo Fijo (IATF) es posible reducir el período de servicio y, en consecuencia, el intervalo entre nacimientos, mejorando así la eficiencia reproductiva del rebaño. Se seleccionaron diez granjas familiares en el municipio de Conceição do Araguaia, en el sureste de Pará, a 991 km de la capital del estado. Se palparon cien vacas cruzadas / multíparas de baja producción para confirmar vacíos y al menos 45 días de parto, referencia basada en las anotaciones encontradas en los cuadernos en las propiedades. El día 0 (implante de progesterona intravaginal y $2 \mathrm{ml}$ de benzoato de estradiol intramuscular), día 8 (retirada del implante más $2.5 \mathrm{ml}$ de prostaglandina, $1.5 \mathrm{ml}$ de gonadotropina coriónica equina y $0.5 \mathrm{ml}$ de cipionato de estradiol, intramuscular), día 10 la inseminación. El diagnóstico de preñez se realizó 60 días después de la inseminación con ultrasonido, las vacas diagnosticadas vacías fueron enviadas a toros con sus respectivas granjas. La tasa de concepción se basó en 94 vacas, ya que 03 vacas murieron y 03 fueron sacrificadas antes del diagnóstico de preñez. Se obtuvo una tasa de concepción del 53\% (50/94), de los cuales el 60\% (30/50) fueron paridos entre 45 a 60 días, mientras que el $40 \%$ (20/50) fueron paridos durante más de 120 días. Se puede concluir que el TAI reduce el período de servicio y, en consecuencia, el intervalo entre nacimientos, lo que también demuestra utilidad en los rebaños agrícolas familiares, lo que puede dar lugar a una mayor posibilidad de producción de leche / ternero / año.

Palabras clave: período de servicio, embarazo, reproducción

\section{Introdução}

A utilização de técnicas avançadas que visem à redução de mão de obra, custos e aumentem a eficiência reprodutiva dos rebanhos, é um dos fatores que mais influenciam para se chegar a elevados índices reprodutivos na pecuária leiteira (Bastos et al., 2003; Quirino et al., 2001; Rigolon et al., 2009).

Devido a problemas cada vez mais frequentes de detecção de cio e queda nas taxas de concepção (TC) em vacas leiteiras, o intervalo entre partos (IEP) tem sido cada vez mais prolongado (Ereno et al., 2007; Ferreira, 2002). De acordo com Hafez \& Hafez (2004) e Ball \& Peters (2006) os principais prejuízos causados por um longo intervalo de partos, são expressos por queda na produção de leite e diminuição do número de crias ao longo da vida produtiva da fêmea bovina. Deveríamos ter sempre em mente que a cada mês que se reduz o intervalo de partos, aumenta-se $8,3 \%$ na produção de leite.

O período de serviço (PS) é o tempo decorrido entre o parto e a concepção, o intervalo médio desejado é de 85 a 115 dias (Pereira et al., 2010; Perotto et al., 2010). Quando esse período ultrapassa 100 dias, cada dia a mais representa gastos na ordem de US\$2,50 a 6,00, dependendo dos custos de produção, do leite e da produtividade do animal (EMBRAPA - PECUÁRIA SUDESTE, 2010). Com a 
inseminação artificial em tempo fixo (IATF) é possível diminuir o IEP (o ideal seria de 12 meses), aumentar a eficiência produtiva e reprodutiva (Ferreira et al., 2013; Gottschall \& Silva, 2012). O objetivo deste estudo foi demonstrar a produtores da agricultura familiar que através da IATF é possível reduzir o PS e consequentemente o IEP, melhorando assim a eficiência reprodutiva do rebanho.

\section{Material e métodos}

O projeto foi desenvolvido durante o período de setembro/2013 a dezembro/2014, aprovado pelo Edital 07/2013 - PROPPG - IFPA. Foram selecionadas 10 propriedades da agricultura familiar, no município de Conceição do Araguaia, Pará, sudeste paraense, distante $991 \mathrm{~km}$ da capital do estado, onde os produtores participantes comprovaram ter experiência na atividade leiteira. Os produtores possuíam entre 20 e 30 vacas no rebanho, além de infraestrutura mínima em suas propriedades como: curral em bom estado, bom suporte forrageiro e uma produção de leite intermitente durante todo o ano. As 100 vacas selecionadas caracterizavam-se como mestiças de baixa produção, multíparas, sem histórico de enfermidades reprodutivas, recebem sal mineral conforme categoria e recomendações dos fabricantes. Todas as vacas eram palpadas para confirmação de vazias e com pelo menos 45 dias de paridas, sendo esta última referência baseada nas anotações encontradas em cadernos nas propriedades. O protocolo utilizado foi o de três passagens: No Dia 0 (implante intravaginal de progesterona e $2 \mathrm{ml}$ de benzoato de estradiol intramuscular), Dia 8 (retirada do implante mais 2,5 $\mathrm{ml}$ de prostaglandina, $1,5 \mathrm{ml}$ de Gonadotrofina Coriônica Equina e $0,5 \mathrm{ml}$ de cipionado de estradiol, intramuscular) e Dia 10 a inseminação.

O diagnóstico de gestação foi feito aos 60 dias após a inseminação com equipamento de ultrassom veterinário Mindray DP2200, pelo mesmo Médico Veterinário. As vacas diagnosticadas vazias foram encaminhadas ao repasse com touros das respectivas propriedades.

\section{Resultados e discussão}

As cem (100) vacas do planejamento da pesquisa foram protocoladas, mas não foi possível selecionar $\operatorname{dez}(10)$ vacas/produtor, mostrando produtor que conseguiu disponibilizar somente três vacas; enquanto outro disponibilizou dezoito vacas (Figura 1). Assim, percebemos a necessidade de flexibilização, quanto ao número de vacas/produtor, quando a pesquisa for direcionada a pequenos produtores.

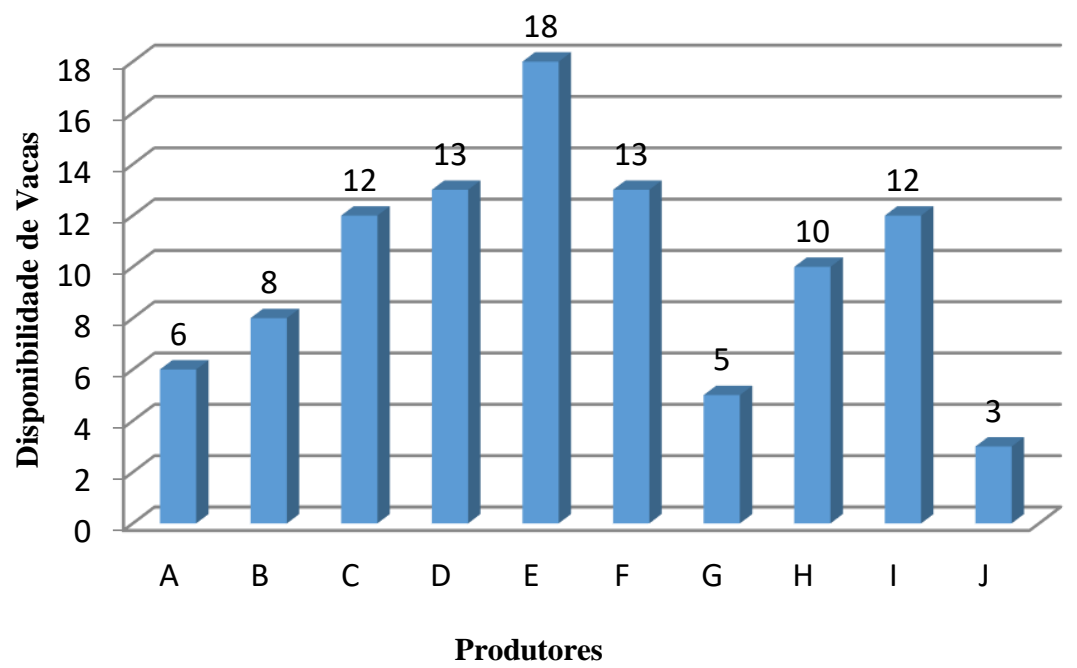

Figura 1. Quantidade de vacas disponibilizada por produtor.

Esta diferença encontrada entre os produtores, em disponibilizar vacas vazias para execução do projeto, foi possível encontrar produtor que só tinha três vacas vazias enquanto outro tinha dezoito vacas vazias, demonstra uma inadequada composição do rebanho leiteiro. Para Campos et al. (2006) 83\% das vacas, em um plantel leiteiro, devem estar em lactação. Sendo que esta inadequada composição do rebanho citada pode ter sido resultado das anotações realizadas por cada produtor, a escrituração zootécnica, por serem dados pouco confiáveis devido não existir rotina em sua realização. A escrituração 
zootécnica é uma ferramenta necessária na bovinocultura de leite (Borges et al., 2011). Para se medir a eficiência dos sistemas de produção utiliza-se a escrituração zootécnica onde sua ausência é sinônimo de problemas (Reis et al., 2017).

A taxa de concepção (Figura 2) foi contabilizada baseada em noventa e quatro (94) vacas, já que três vacas vieram a óbito e três foram encaminhadas ao abate antes do diagnóstico de gestação. Obteve-se uma taxa de concepção de 53\% (50/94), destas, 60\% (30/50) estavam paridas entre 45 a 60 dias, enquanto $40 \%$ (20/50) estavam paridas a mais de 120 dias.

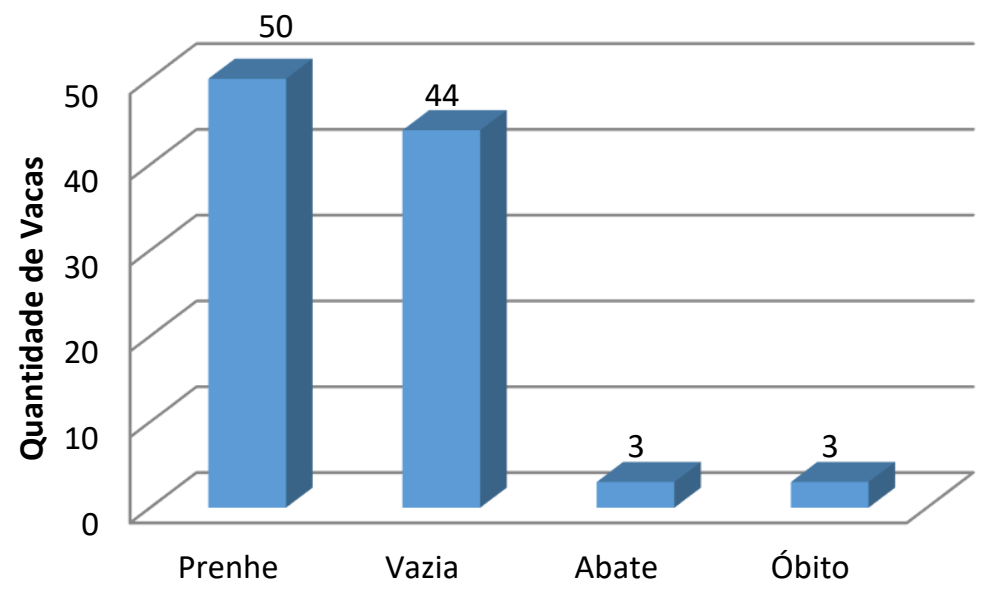

Figura 2. Taxa de concepção da IATF de 53\% (50/94). Quantidade de vacas prenhes, vazias, encaminhadas ao abate ou que morreram por enfermidade não diagnosticada.

A taxa de concepção (53\%) encontrada nesta pesquisa está acima das taxas encontradas pelo Grupo GERAR - Grupo Especializado em Reprodução Aplicada ao Rebanho (2017), em bovinos de leite. As taxas encontradas pelo grupo foram nulíparas (39,5\%), primíparas e multíparas (34,3\%), e intermediárias as taxas encontradas por Santos \& Sousa (2014) que obtiveram taxas de 65\% de vacas prenhas na fazenda A onde a média era 5 litros/vaca/dia e na fazenda B foram 34,2\% de vacas gestantes, sendo que a média destas era de 15 litros/vaca/dia. Sugere-se então que a taxa de 53\% encontrada nesta pesquisa pode estar relacionada com a produtividade baixa dos animais utilizados.

Foi encontrada uma média de 60 dias para PS e 12 meses de IEP nas vacas que estavam paridas entre 45 a 60 dias e 130 dias para PS e 14 meses de IEP nas vacas que estavam paridas a mais de 120 dias. Em estudos realizados com rebanhos mestiços holandês x zebu, Araújo Neto et al. (2002) observaram um intervalo de partos de 14,3 meses; Facó et al. (2002) 407,5 dias, aproximadamente 13,6 meses, semelhante as observações de Freitas et al. (2002) que descrevem uma média de 412,7 dias (13,7) meses. Desta maneira a média para o IEP encontrado, 12 e 14 meses, está em conformidade com os autores citados, evidenciando assim o que a IATF pode agregar em rebanhos bovinos da agricultura familiar, pois para as vacas que obtiveram a média de 130 de PS foi possível ajustar sua vida reprodutiva e para as demais, foi possível mantê-las dentro das recomendações sobre manejo reprodutivo. O intervalo médio desejado para PS é de 85 a 115 dias (EMBRAPA - PECUARIA SUDESTE, 2010).

\section{Conclusão}

Conclui-se que a IATF reduz o período de serviço e consequentemente o intervalo entre partos em rebanhos bovinos da agricultura familiar, podendo resultar em maior possibilidade de produção/ano de leite e bezerros.

\section{Referências bibliográficas}

Araújo Neto, R. B. de., Magalhães, J. A., Leal, J. A., Nascimento, M. P. S. B. C., Nascimento, H. T. S., Lopes, E. A.., Rodrigues, B. H. N., Leal, T. M., Italiano, E. C., Lima, V. M. B. (2002). Coeficientes técnicos. Brasília, DF: Embrapa. Disponível em: <http://sistemasdeproducao.cnptia.embrapa.br/FontesHTML/Leite/LeiteMeioNorte/ coeficientes.html>. Acesso em: 30 jul. 2013. 
Ball, P. J. H. \& Peters, A. R. (2006). Reprodução em bovinos. São Paulo, São Paulo, Brasil: Roca.

Bastos, G. D. M., Gonçalves, P. B. D., Machado, M. S. N., Restle, J., Neves, J. P., Oliveira, J. F. C., . . . Faturi, C. (2003). Indução hormonal da ovulação e desmame precoce na fertilidade pós-parto de vacas de corte homozigotas e heterozigotas para o microssatélite BMS3004. Revista Brasileira de Zootecnia, 32(5):1093-1103.

Borges, M. S., Guedes, C. A. M. \& Assis, R. L. (2011). Um estudo do "projeto balde cheio" como vetor do desenvolvimento sustentável do pequeno produtor de leite. Revista Brasileira de Agropecuária Sustentável, 1(1):151-161.

Campos, A. T., Moraes, F. A. \& Leite, E. G. (2006). Composição do rebanho e sua importância no manejo: Embrapa Gado de Leite.

Ereno, R. L., Barreiros, T. R. R., Seneda, M. M., Baruselli, P. S., Pegorer, M. F. \& Barros, C. M. (2007). Taxa de prenhez de vacas Nelore lactantes tratadas com progesterona associada à remoção temporária de bezerros ou aplicação de gonadotrofina coriônica eqüina. Revista Brasileira de Zootecnia, 36(5):1288-1294.

Facó, O., Martins Filho, R. \& Lôbo, R. N. B. (2002). Análise do desempenho reprodutivo de cinco grupos genéticos Holandês x Gir no Brasil. Paper presented at the Embrapa Caprinos e OvinosArtigo em anais de congresso.

Ferreira, A. M. (2002). Como reduzir o intervalo entre partos. DBO Especial Mundo do Leite, 132-33.

Ferreira, M. C. N., Miranda, R., Figueiredo, M. A., Costa, O. M. \& Palhano, H. B. (2013). Impacto da condição corporal sobre a taxa de prenhez de vacas da raça nelore sob regime de pasto em programa de inseminação artificial em tempo fixo (IATF). Semina: Ciências Agrárias, 34(4):1861-1868. doi: http://dx.doi.10.5433/1679-0359.2013v34n4p1861.

Freitas, M. S., Cavalcanti, H., Costa, C. N., Freitas, A. F., Torres, R. A., Rennó, F. P. \& Araúlo, C. V. (2002). Idade ao primeiro parto, intervalo de partos, produção na primeira lactação e produção por dia de intervalo de partos de vacas girolando. Reunião Anual da Sociedade Brasileira de Zootecnia,, 39.

Gottschall, C. S. \& Silva, L. R. (2012). Resposta reprodutiva de novilhas de corte aos dois e três anos de idade submetidas a diferentes protocolos para inseminação artificial em tempo fixo (IATF). Veterinária em Foco, 10(1):16-25.

Hafez, B. \& Hafez, E. (2004). Reprodução Animal (Vol. 1, pp. 513): Manole: São Paulo, Brasil.

Pereira, E. S., Pimentel, P. G., Queiroz, A. C. \& Mizubuti, I. Y. (2010). Novilhas leiteiras (Vol. 1). Fortaleza, Ceará: Graphiti Gráfica e Editora Ltda.

Perotto, D., Kroetz, I. A. \& Rocha, J. L. (2010). Milk production of crossbred Holstein $\times$ Zebu cows in the northeastern region of Paraná State. Revista Brasileira de Zootecnia, 39(4):758-764.

Quirino, C. R., Salvador, D. F., Andrade, V. J., Vale Filho, V. R., Ribeiro Filho, A. L., Nogueira, L. A. G. \& Gattass, C. (2001). Eficiencia reprodutiva de femeas Nelore sincronizadas com o protocolo Crestar, acasaladas com touros previamente selecionados pela classificacao andrológica por pontos (CAP) e libido. Revista Brasileir de Reproduçõ Animal, 25(3):304-307.

Reis, E. M. B., Vieira, J. A., Demeu, M. A. L. F. A. \& Bruhn, F. R. P. (2017). Identificação de pontos fracos e fortes associados à qualidade do leite em propriedade leiteiras de agricultura familiar. PUBVET, 11840-946.

Rigolon, L. P., Prado, I. N., Cavalieri, F. L. B., Nascimento, W. G., Copovila, L. C., Ramos, F. S. \& Moreira, F. B. (2009). Effect of the dry matter intake level on the sanguine profile of glucose, insulin, urea, estrogen and progesterone and concentration of IGF-I in the follicular liquid of crossbred heifers. Brazilian Archives of Biology and Technology, 52(1):61-68.

Santos, I. I. \& Sousa, I. L. G. (2014). Relação da taxa de gestação utilizando inseminação artificial em tempo fixo (iatf) com a taxa de produção de leite. Saber Digital, 138-51.

Recebido: 2 de agosto, 2019.

Aprovado: 1 de novembro, 2019.

Publicado: 29 de fevereiro, 2020.

Licenciamento: Este artigo é publicado na modalidade Acesso Aberto sob a licença Creative Commons Atribuição 4.0 (CC-BY 4.0), a qual permite uso irrestrito, distribuição, reprodução em qualquer meio, desde que o autor e a fonte sejam devidamente creditados. 